\title{
Nanophotonic Metasurfaces for Biosensing and Imaging
}

\author{
Hatice Altug ${ }^{1}$ \\ ${ }^{1}$ EPFL, Ecole Polytechnique Federale de Lausanne, School of Engineering, Lausanne, Switzerland, 1015 \\ https://bios.epfl.ch/
}

\begin{abstract}
Nanophotonics excels at confining light into nanoscale optical mode volumes and generating dramatically enhanced light matter interactions. These unique aspects have been unveiling a plethora of fundamentally new optical phenomena, yet a critical issue ahead for nanophotonics is the development of novel devices and applications that can take advantage of these nano-scale effects. It is expected that nanophotonics will lead to disruptive technologies in energy harvesting, quantum and integrated photonics, optical computing and including biosensing. To this end, our research is focused on the application of nanophotonics to introduce powerful biosensors that can have impact on a wide range of areas including basic research in life sciences, early disease diagnostics, safety and point-of-care testing. In particular, we exploit nanophotonics and its integration with microfluidics to address key challenges of current biosensors and develop devices that can enable label-free, ultra-sensitive, multiplexed, rapid and real-time measurements on biomolecules, pathogens and living systems. In this talk I will present some of our recent work on nanophotonic metasurfaces for biosensing and bioimaging as well as their applications in real-world settings.
\end{abstract}

\section{Introduction}

With the emerging new healthcare initiatives including personalized medicine, global health care and point-ofcare diagnostics, we are witnessing a paradigm shift in the way medical care is provided. These initiatives are demanding next generation diagnostics and bioanalytical tools that can deliver a long list of desired specifications such as high sensitivity, multiplexing, real-time operation, and miniaturization, low-cost. Nanophotonics offers unique opportunities to meet such requirements. To this end the effort of our lab is focused on using nanophotonics to develop powerful biosensors can impact numerous fields ranging from basic research in life sciences, early disease diagnostics, personalized medicine and point-ofcare testing to environmental/food monitoring and safety. In particular we employ a variety of nanophotonic techniques including dielectric metasurfaces and plasmonics to introduce fundamentally new optical detection schemes. We exploit electromagnetic spectrum spanning from visible to mid-infrared wavelengths for developing biosensors based on optical refractive index variations and surface enhanced IR absorption spectroscopy. We integrate our sensors with microfluidic systems for efficient analyte trapping, sample manipulation and automation. We also combine the use of smart data science tools with bioimaging based sensing architectures for achieving unprecedented performance metrics. In this talk, I will present some of our recent work on nanophotonic metasurfaces for biosensing and bioimaging and show examples how they can be used in real-world applications [1-14].

\section{Nanophotonic Biosensors and Imaging Systems}

\subsection{Mid-IR Plasmonics for Label-Free, Sensitive and Real-Time Monitoring of Biomolecular Conformational Changes}

We use engineered Mid-IR plasmonic antennas to overcome fundamental limitations of conventional IR spectroscopy by surface enhanced infrared absorption (SEIRA) spectroscopy method. Recently, we showed a plasmonic metasurface consisting of multi-resonant nanoantennas for simultaneously distinguishing multiple analytes in heterogeneous biological samples [2]. We demonstrated that this device can spectroscopically resolve the interaction of biomimetic lipid membranes with peptides as well as the dynamics of synaptic vesical cargo release. Our lab has been also advancing label-free spectroscopic identification of secondary structure conformation of nanometric thin protein layers in aqueous solution. Detection of protein conformations is immensely important to understand and diagnose incurable diseases resulting from protein misfolding such as Parkinson's and Alzheimer's disease. In our 2017 work, we used $\alpha$-synuclein, a protein associated with plaque formation in Parkinson's diseases [3]. By leveraging plasmonically enhanced amide-band proteins, we were able to measure a random coil to cross $\beta$-sheet conformational change as a result of $\alpha$-synuclein aggregation. We showed high structural sensitivity of our

\footnotetext{
*Corresponding author: hatice.altug@epfl.ch
} 
biosensor by distinguishing the characteristic $\beta$-sheet conformations between a native protein and pathological fibrillar aggregates. In a recent work, we presented the first experimental demonstration of real-time label-free spectroscopic monitoring of secondary structure changes in a protein monolayer in aqueous solution [4]. We have been contributing to the fundamental understanding of SEIRA and determining its limitations. Since SEIRA is a new field, the general notion on sensitivity parameters is still not well developed. In a recent work, we introduced methodologies in order to quantify the limit of detection which can provide a valuable reference points for determining biosensor performance metrics [5].

\subsection{All-Dielectric Metasurfaces for Biosensensing and Imaging}

All-dielectric metasurfaces are fast emerging in nanophotonics field due to their low intrinsic loss, strong resonance enhancement of both electric and magnetic near-fields and complementary metal-oxide semiconductor (CMOS) compatibility. In 2018 [6], we introduced a nanophotonic method capable of detecting mid-infrared molecular fingerprints without the need for spectrometry, frequency scanning, or moving mechanical parts. Our method leverages a 2D array of high-Q dielectric metasurface pixels designed to provide strong near-field enhancement, where the resonance positions of individual metapixels are varied over a target mid-IR fingerprint range. Significantly, we assign each resonance position to a specific pixel of the metasurface, establishing a one-to-one mapping between spectral and spatial information. This unique configuration enables to sample near-field enhanced molecular absorption signatures of surface adsorbed molecules at multiple spectral points and to translate this information into a $2 \mathrm{D}$ barcode-like spatial absorption map for imaging-based detection. Recently we developed metasurfaces operating in visible spectrum, and by utilizing hyperspectral imaging and smart data science tools we are currently investigate how to unleash the full potentials of high-Q dielectric metasurfaces for achieving biosensors with unprecedented performance metrics [Yesilkoy et al. accepted 2019].

\subsection{Opto-Fluidic Biosensors for Real-Time Secretion Analysis from Live Cells and Single Cells}

Our lab is also working on the integration of nanophotonics and microfluidics to develop biosensors that can analyze live cells at the single cell resolution. In 2017, we introduced a nanophotonic biosensor enabling real-time monitoring of live cell secretions in a label-free manner [7]. Our microfluidic-integrated biosensor, compatible with common inverted microscopes can able to distinguish cell secretion events during long-term live cell culture. In 2018, we demonstrated a new technology for label-free monitoring of cell responses at the single cell sensitivity [8]. The device enables long term monitoring of isolated individual cells and identifying their secretion activity in real time, all without disturbing the cell environment or introducing invasive agents.

\section{References}

1. Oh and Altug "Performance Metrics and Enabling Technologies for Nanoplasmonic Biosensors", Nature Communications Vol. 9, p. 5263 (2018).

2. Rodrigo et al., "Resolving Molecule-Specific Information in Dynamic Lipid Membrane Processes with Multi-Resonant Infrared Metasurfaces", Nature Communications Vol. 9, p. 2160 (2018).

3. Etezadi et al., "Nanoplasmonic Mid-Infrared Biosensor for in-vitro Protein Secondary Structure Detection" Light Science and Application Vol.6 e17029 (2017).

4. Etezadi et al., "Real-Time in-Situ Secondary Structure Analysis of Protein Monolayer with MidInfrared Plasmonic Nanoantennas", ACS Sensors Vol. 3, p. 1109-1117 (2018).

5. John-Herpin et al. "Quantifying Limits of Detection of Surface-Enhanced Infrared Spectroscopy with Grating Order-Coupled Nanogap Antennas", ACS Photonics Vol. 5, p.4117-4124 (2018).

6. Tittl et al., "Imaging-Based Molecular Barcoding with Pixelated Dielectric Metasurfaces", Science Vol. 360, p. 1105-1109 (2018).

7. Li et al., "Plasmonic Nanohole Array Biosensor for Label-Free and Real-Time Analysis of Live Cell Secretion", Lab on a Chip Vol. 17, p. 2208-2217 (2017).

8. Li et al., "Label-Free Optofluidic Nanobiosensor Enables Real-Time Analysis of Single-Cell Cytokine Secretion", Small Vol. 14, 1870119 (2018).

9. Beluskin et al., "Nanoparticle-Enhanced Plasmonic Biosensor for Digital Biomarker Detection in a Microarray", ACS Nano Vol. 12, p. 4453-4461 (2018).

10. Yesilkoy et al. "Phase-Sensitive Plasmonic Biosensor Using a Portable and Large Field of View Interferometric Microarray Imager", Light Science and Application Vol. 7, p. 17152 (2018).

11. Soler et. al "Two-Dimensional Label-Free Affinity Analysis of Tumor Specific CD8 T Cells with a Biomimetic Plasmonic Sensor", ACS Sensors Vol. 3, p. 2286-2295 (2018).

12. Mohammadi et al. "Nanophotonic Platforms for Enhanced Chiral Sensing", ACS Photonics Vol, p. 2669-2675 (2018).

13. Gupta et al. "Self-assembly of nanostructured glass metasurfaces via templated fluid instabilities" Nature Nanotechnology (2019).

14. Rodrigo et al., "Mid-Infrared Plasmonic Biosensing with Graphene" Science Vol 349, pp. 165-168 (2015). 